\title{
GEOMETRY: OUR CULTURAL HERITAGE
}

\author{
REVIEWED BY H. WU
}

\author{
Geometry: Our Cultural Heritage \\ Audun Holme \\ Springer 2002. 378 pages.
}

Notices Amer. Math. Soc., 51 (2004), 527--535.

Audun Holme has written an elementary book on geometry, hereafter referred to as [Holme], aimed primarily at American mathematics teachers in both elementary and high school, as well as at "an informed public interested in a making a new beginning in mathematics". The book is divided into two parts: Part I, entitled "A Cultural Heritage", and Part II, "Introduction to Geometry". The preface outlines two possible college courses to be given according to this division: a course based on Part I for prospective elementary school teachers and students who are interested in acquiring some mathematical culture, and a second course based on Part II which, one may infer from the stated prerequisites, is designed primarily for prospective high school teachers.

The average teacher's deficiency in mathematical knowledge is wellknown. ${ }^{1}$ Fortunately, it is no longer controversial in year 2004 to assert that a remedy must begin with better mathematics instruction for prospective elementary teachers. How much Part I of [Holme] can contribute to this effort will be the subject of the first section. In the second section, we shall examine Part I from the point of view of a student who wants to learn some geometry for historical or cultural reasons. Part II will be reviewed separately in the third and final section where, in addition to some comments on the success of Part II as a cross between a technical textbook and a "geometry-appreciation" text, considerable space will be devoted to a discussion of the state of

Date: January 31, 2004.

I am extremely grateful to Allyn Jackson and Ralph Raimi for their invaluable help in the preparation of this article.

${ }^{1}$ This deficiency will be described at some length later in this review, but given the present politically charged climate in mathematics education, let me hasten to add that teachers themselves are not the sole cause of this deficiency. Inadequate mathematics instruction in universities and low salaries in the teaching profession also have a lot to do with it. 
high school geometry. The latter is in fact one of the three most notorious problems in school mathematics education, the other two being the teaching of fractions in upper elementary school and the teaching of beginning algebra in middle school. While this is my personal opinion, there is little doubt that the teaching of high school geometry, which is tantamount to the teaching of mathematical proofs in schools, is worthy of some attention from the mathematics community.

Before we proceed further, a caveat is in order. Mathematics teachers in different countries have different (professional) cultures and distinctly different needs. It is therefore important to note that [Holme] has in mind American teachers. This can be seen in the preface, where the author refers to the public's "math avoidance" and "math anxiety", and suggests using this book in a "Community College". The first and third sections of this review therefore explicitly refer to American teachers.

\section{Part I as Professional Development}

The preface of [Holme] deplores the teaching of geometry to prospective teachers as "pedantic and formalistic". Part I is offered as an alternative. The fact that elementary teachers need help in terms of content knowledge is hardly news (cf. the report [NCT]), but before rushing to the patient with remedy in hand, we would do well to first make a correct diagnosis. What exactly do elementary teachers need to know in geometry? At least part of an answer can be inferred from [Beckmann] or [Wu-Braxton]. In terms of specific technical knowledge, they first need a firm grasp of the core ideas of geometric measurements because length, area, and volume are the staples of elementary school geometry. For example, most elementary teachers have difficulty explaining what the length of a circle (i.e., circumference) means, and too often, area means no more than "length times width". The far-from-trivial task facing them is to learn to explain these concepts in a mathematically viable fashion without any knowledge of the Riemann integral or the Lebesgue measure. They also need to learn, urgently, precise definitions of key concepts as well as the importance of having precise definitions. Too many of them were taught mathematics using intuitive definitions rather than mathematically correct ones. Thus most elementary teachers would have difficulty explaining what a polygon is other than "a closed plane figure with straight sides", or what is meant by two figures being congruent other than "same size and same shape", or when two figures are similar other than that they have the "same shape but not the same size". How to use definitions appropriately 
in the mathematics classroom is a nontrivial issue in mathematics education that cannot be disposed of in a few sentences in this review, but $[\mathrm{Wu} 4]$ would serve as a starting point for such a discussion. See especially pp. $2-4$ and $\S \S 2-3$ of that article. A third area of weakness in elementary teachers' geometric equipment is the inability to explain why something is true. This means, at least, that the idea of proving theorems does not come naturally to them. The mathematics of elementary school is not predominantly about theorem-proving, so I am not talking about anything fancy such as a proof of why the three altitudes of a triangle meet at a point. What I have in mind is rather the difficulty of elementary teachers in producing explanations of basic, simple geometric facts such as the Pythagorean Theorem or the sum of the angles of a triangle being $180^{\circ}$.

Above and beyond technical skills, most elementary teachers do not possess the right disposition towards geometry. To many, geometry is a foreign concept, to the point that some of them literally do not know what to do with a ruler and compass when asked to draw a circle or connect two points by a straight line. It goes without saying that, as a consequence, they have almost no geometric intuition. Even among those teachers who know that there is a circle passing through any three noncollinear points, for example, rare is the person who who can draw a rough mental picture of this circle when three points are explicitly given. Overcoming such apathy, sometimes even phobia, relative to geometry requires new thinking and new instructional strategies.

With all this in mind, suppose you want to offer elementary teachers a new beginning in geometry. Your decision is to give them an abbreviated version of the history of Euclidean geometry assuming only a knowledge of school mathematics. So you go from Thales of Miletus (circa 600 B.C.) to Euclid, Eudoxus, Archimedes, Apollonius, down to Hypatia (d. 415 A.D.). You keep them riveted in the historical details of the Roman Empire, thereby providing the thread that connects the death of Archimedes to the saga of the Alexandrians (Ptolemy, Heron, Diophantine, and Pappus) all the way to the murder of Hypatia. Then you paint a picture of the intellectual poverty of the Dark Ages before picking up the geometric thread again with an account of Desargues, Pascal and Descartes. For the sake of variety, you also throw in the briefest discussion possible of algebraic varieties, fractals and catastrophe theory, even if you must know in your heart of hearts that, in such a condensed form, none of it would be understood.

The content of the preceding paragraph is in fact a synopsis of Part I of [Holme]. Would learning this material make elementary teachers 
teach geometry better? It would no doubt make them aware of some interesting information, and a few might even be sufficiently stimulated to learn more about the subject. But it would be difficult to argue that much of this material is relevant to their everyday classroom needs.

For a long time, dating back at least to the days of the New Math, mathematicians' attitude towards the teaching of prospective teachers has been encoded in the generic phrase, "teach them more mathematics". Few until recent times have asked the obvious follow-up question: "Are we teaching them the kind of mathematics that we consider interesting and important, or are we teaching them mathematics that has a direct bearing on the school classroom?" ([Wu1], [Wu3]; subsequently elaborated in [MET].) These two kinds of mathematics are not the same (ibid.), and the failure to heed the difference between the two is a major reason why we have mathematically unprepared teachers. Part I of [Holme] contains information that is interesting and ultimately important for making elementary teachers well-rounded teachers of geometry, but it does little to help them understand the basic geometric concepts or learn the necessary technical skills they need to better teach their students.

The disregard of the relevance to the school mathematics classroom unfortunately spills over into in-service professional development as a whole. In the name of teaching "content-rich" mathematics to teachers, many topics of elementary discrete mathematics, for example, are foisted upon teachers while basic bread-and-butter issues such as fractions, decimals, area, etc., are left unattended (cf. [Wu2], Sample 1 and especially Sample 2). Thus in more ways than one, the mathematics community has let teachers down.

Mathematicians naturally cannot assume the responsibility for the downfall of mathematics teachers because the education of these teachers is the basic charge of the schools of education across the land. All the same, it is regrettable that, for so long, the mathematics community has neglected such a vital part of its service to society at large.

\section{PART I AS GeOMETRY-Appreciation}

If we ignore the issue of professional development, Part I of [Holme] can be taken on its own terms as a non-technical introduction to geometry and its history. As such, it presents an enjoyable romp through the history of elementary geometry (roughly up to projective geometry), much like what E.T. Bell did in Men of Mathematics ([Bell]) for the history of mathematics. Unlike Bell though, Holme is more honest

about putting good story-telling above historical accuracy (cf. lines 1-5 
of p. vi). Part I also calls to mind two other semi-popular accounts of geometry, Heilbron's Geometry Civilized: History, Culture, and Technique ([Heilbron]) and the Geometry and the Imagination of Hilbert and Cohn-Vossen ([Hilbert-CV]). The difference is that the former is exclusively concerned with Euclid's Elements rather than the historical development issuing from it, and the latter is less about history and more about the mathematical panorama in the field of geometry around 1920. Both of these volumes set high scholarly standards that few books can hope to approach.

The strength of Part I of [Holme] lies in its emphasis on the interaction between mathematical activities and their historical background. This may be the only elementary textbook on mathematics history that describes, sensibly, the lapse of the western world into the Dark Ages. Students who are not aware that the human race existed before 1776 , or that the Bible has historically valid references to Babylonia, would finally learn upon reading Part I that Iraq was in fact the site of a once magnificent civilization to which the whole world still owes a great debt. This in itself would almost justify the writing of [Holme]. Many would also appreciate the description of the possible complicity of St. Cyril (of Alexandria) in Hypatia's death on pp. 122-125; it is as riveting as a good detective novel.

There are, however, quite a few missteps in [Holme] resulting from questionable mathematical or historical judgment. These missteps prevent a whole-hearted recommendation of Part I. The discussion on pp. 17-26 of the famous Babylonian clay tablet Plimpton 322 is a case in point.

This tablet contains a list of four columns and fifteen rows of whole numbers. It is dated to about 1800 B.C. ${ }^{2}$ and was first brought to the world's attention in 1945 by the mathematical historians Otto Neugebauer and Abraham Sachs when they made the spectacular discovery of a Pythagorean triple 3 in every row. For example, the first three rows are (in our usual numeral notation):

$\begin{array}{cccc}0.9834028 & 119 & 169 & 1 \\ 0.9491586 & 3367 & 4825 & 2 \\ 0.9188021 & 4601 & 6649 & 3\end{array}$

\footnotetext{
${ }^{2}$ I have adopted the dating of this tablet in [Robson 1].

${ }^{3}$ Positive integers $a, b, c$ so that $a^{2}+b^{2}=c^{2}$.
} 
Imbedded in these twelve numbers are the three Pythagorean triples

$\begin{array}{ccc}120 & 119 & 169 \\ 3456 & 3367 & 4825 \\ 4800 & 4601 & 6649\end{array}$

Observe, however, that the first number of each triple $(120,3456$, and 4800, resp.) is missing from the list. Why this is so is among the questions that Plimpton 322 raises but which have resisted definitive answers to this day. It is generally agreed that the Babylonians knew the so-called Pythagorean theorem ${ }^{4}$ as well as ways to write down Pythagorean triples. To what extent they possessed such knowledge (e.g., did they know a proof of the Pythagorean theorem, and if so in what form?) may never be known due to the meagerness of the historical record in this period. The absence of certainty naturally sparks scholarly debate.

Into this debate steps the author. He wants to impress on the reader the fact that the Babylonians were in possession of very advanced mathematical knowledge. Not content with the fact that Plimpton 322 already makes this fact abundantly clear, he is intent on going much further. First he tells us that he would "... present the simplest and most beautiful proof I know of [the Pythagorean Theorem]. In all likelihood, this is the Babylonian proof, the proof they knew." (p. 18; the author's italics). The proof in question is the traditional proof that makes use of the following well-known picture and the identity $(a+b)^{2}=a^{2}+2 a b+b^{2}$ :

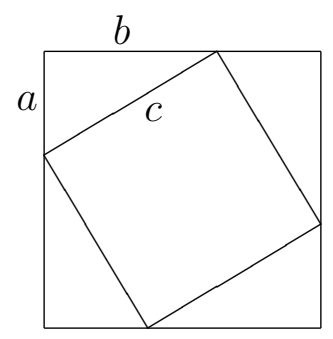

Let it be known right away that there is not even a hint of any historical record in the standard literature that would support such a claim. If the author wants to assert something of such striking novelty, it would be incumbent on him to produce the needed evidence, and this he has not done. Next, he explains that the Pythagorean triples in Plimpton 322 were all generated by a pair of whole numbers $p$ and $q$ in the manner

\footnotetext{
${ }^{4}$ Pythagoras lived about 1200 years after Plimpton 322.
} 
described by the following theorem, which I now quote verbatim from p. 23, including the typography: ${ }^{5}$

Theorem 1 (Ancient Wisdom of Babylon). All primitive Pythagorean triples $(a, b, d)$ are given by

$$
a=q^{2}-p^{2}, b=2 p q, d=q^{2}+p^{2},
$$

where $p$ and $q$ are positive integers, $q>p$, without $a$ common factor $>1$. Moreover, $p$ and $q$ are not both odd numbers.

The primitivity of $(a, b, d)$ refers to the fact that $a, b, d$ have no common factor other than \pm 1 . The author goes on to say that "not only did the Babylonians know 'Pythagoras' Theorem', but they knew the theory of primitive Pythagorean triples as well, and may have used it to compile trigonometric tables for use in their astronomy and engineering". 6

Now, this " $p, q$ theory" of Plimpton 322 (in the terminology of [Robson 1]) has been around for a while. It was first proposed by Neugebauer and Sachs, and it has not lacked for followers. But in the absence of solid evidence, a theory is not a fact, and the implication of certitude by the use of such phrases as "Ancient Wisdom of Babylon" and "they knew the theory of primitive Pythagorean triples" crosses the line of scholarly propriety. In this context, recall that about 1500 years after Plimpton 322 had been written, Euclid asserted in the Elements that if $p, q$ are whole numbers, both even or both odd, and $q>p$, then

$$
\frac{1}{2}\left(q^{2}-p^{2}\right), \quad p q, \quad \frac{1}{2}\left(q^{2}+p^{2}\right)
$$

form (what we call) Pythagorean triples. ([Heath 1], p. 63 of Volume 3, Lemma 1 before Proposition 29 of Book X.) While Euclid provided a full proof as usual, he made no mention of the concept of primitivity. Moreover, he also seemed unaware of the desirability of such an enumeration. What then could lead Holme to conclude that the Babylonians had this kind of mathematical sophistication if not for the desire to impose our mathematical aesthetics on Plimpton 322 at the expense of historical plausibility?

In the last sentence, I have paraphrased part of the conclusion of a remarkable paper by Eleanor Robson, [Robson 1], which re-assesses

\footnotetext{
${ }^{5}$ For a reason which will soon be apparent, I have replaced the author's $u$ and $v$ by $p$ and $q$, resp.

6 In the preface (p. vi), even the last vestige of doubt is banished: “...the astronomers and engineers - or if we prefer, the astrologers and priests - of ancient Babylonia, or Mesopotamia, used these insights to construct trigonometric tables."
} 
Plimpton $322 .{ }^{7}$ She argues most persuasively that, when the historical and mathematical contexts of Babylonia circa 1800 B.C. are taken into account, neither the " $p, q$ theory" of Theorem 1 above nor the trigonometric table theory has much credibility as a satisfactory explanation of Plimpton 322. She goes on to explain why this tablet was most likely a kind of "work sheet" given by a teacher to students to practice finding lengths of the sides of a right triangle by the then-known method of "reciprocal pairs" and "completing the square". Most importantly, what she wrote on p. 202 of [Robson 1] after thirty-six pages of close reasoning is very pertinent to this review:

[The explanation of reciprocal pairs] is perhaps as far as we can go on present evidence: without closer parallels we run the risk of crossing the fuzzy boundary from history to speculation. The Mystery of the Cuneiform Tablet has not yet been fully solved.

Such a caveat would have made the [Holme] discussion of Plimpton 322 both more accurate and more interesting.

Due to the lack of space, I will content myself with the barest mention of three more examples of excess in [Holme]. The disparaging comment about Bourbaki on p. 17 should have been counter-balanced by a reference to something like [Borel]. The attempt (on p. 121) to put Theon of Alexandria and Hilbert on the same footing as mathematicians is shocking, coming as it does from a professional mathematician. Finally, elevating Hypatia to "one of the greatest names within philosophy, mathematics, and other sciences" (also on p. 121) seems to be nothing but political correctness spinning out of control (cf. [Deakin 1], [Deakin 2]).

It remains to endorse Holme's decision to insert some mathematical details into the general discussion of Part I, but to part company with him on the poor execution of such a good idea. A little more consideration for the readers' technical limitations would have suggested making every definition as simple as possible. The definition of a regular polyhedron on p. 58 as a convex polyhedron with congruent regular polygons as faces, for example, includes the additional requirement that all the "polyhedral angles" at the vertices are "congruent". But the concept of a polyhedral angle is too sophisticated for the average reader, and

\footnotetext{
7 [Robson 2] is a popular version of [Robson 1] and the former is what most mathematicians would likely get to read about Robson's work. For many reasons, I urge you to read the original article [Robson 1] because [Robson 2] does not do [Robson 1] justice.
} 
the few cryptic phrases on p. 57 on the "configuration" of a polyhedral angle reveal little about this concept, much less qualify as a definition. Why not just define a regular polyhedron to be a convex polyhedron with faces which are regular polygons of a fixed number of sides so that the number of edges emanating from each vertex is a constant, and add:

It can be shown from this definition, but at the cost of some effort, that at each vertex of a regular polyhedron, the polyhedron looks exactly the same in a sense that can be made precise.

This at least would have the virtue of being correct in addition to giving the reader an equal amount of information (if not more). And was the author aware that what he calls "a very simple and conceptual proof" of Cramer's rule on pp. 141-144 actually requires the use of the inverse of a matrix and sophisticated abstract reasoning to justify (see lines $5-8$ on p. 144)? There are many other examples in Part I which likewise overtax a reader's mathematical capabilities.

\section{REVIEW OF PART II}

Until someone can convincingly demonstrate that there is something better to teach high school geometry students, Euclidean geometry (in its many guises) will continue to be the mainstay. Yet, in spite of the fact that Euclid has never left the classroom for the last twenty-three centuries, he has not always fared well with students. Some of the older generations may have fond memories of proofs in geometry, and many became mathematicians because of their exposure to Euclid. See, for example, the ecstatic account in [Russell], p. 33, or the charming article [Osserman]. But there is also a dark side of this relationship between Euclid and geometry students, and for that one has to look into the modern school classroom.

A very rough estimate is that between 1970 (if not earlier) and 1990, theorem-proving in high school geometry often degenerated into a ritual devoid of mathematical content. Some well-intentioned educators and teachers reacted to this deterioration by declaring that, if students don't learn proofs, then proofs do not belong in the classroom. Geometry courses would henceforth consist of what is known as "hands-on activities" sans proofs, and the book [Serra] serves to give some idea of 
what this means. ${ }^{8}$ At a meeting in Washington D.C. in March of 2003, when a professional developer from the Midwest found out that the exam for the credentialing of California's high school teachers requires the writing of proofs in geometry, he remarked on how unfair this would be for teachers in his own state. (Proofs are no longer taught in most high schools in that region.)

The article "When good teaching leads to bad results ..." of [Schoenfeld], dated 1988, gives a hint of what went wrong after 1970. Schoenfeld observed twelve mathematics classes in a high school and recorded in detail his observations of a 10th-grade geometry class which, one may assume, was typical among the twelve. Here is a summary of what took place in that classroom: Proofs had to be written down in the two-column format and no other format was accepted; only proofs of trivial theorems were emphasized, and consequently geometric instruction dwindled to a meaningless ritual of writing down a sequence of steps in accordance with a particular grading policy rather than about the discovery of proofs of theorems; the focus of straightedge-compass constructions was on the procedural correctness and the pictorial accuracy rather than on the geometric reasoning behind the construction. And so on.

A mathematician reading [Schoenfeld] would be struck by the stunning lack of basic understanding of mathematics implicit in every bad practice described therein. Until teachers know a little more mathematics in general, and a little more geometry in particular, these disasters will continue to overwhelm mathematics classrooms. A natural question is why any educational system would consider this kind of teaching to be good teaching (see lines 8-15 on p. 162 of [Schoenfeld]). To the extent that the main goal of mathematics education is to improve learning in the school classroom, the emergence of such a system must be regarded as an exceedingly alarming trend. Did subsequent education research respond to [Schoenfeld] by making a a vigorous probe into how such a cancerous growth could develop in the system? Did the teacher qualification problem, so mercilessly exposed, spur the education establishment into taking concrete steps for correction? Apparently not. ${ }^{9}$

But to go back to the bad practices described in [Schoenfeld], a fundamental issue is that many teachers feel lost in a geometric environment. Without any intuition to help them navigate the smallest steps in the geometric terrain, they are forced to reduce everything to

\footnotetext{
${ }^{8}$ It offers heuristic arguments, and only heuristic arguments, for the first 676 page of this 830 page tome. A set of (badly mangled) geometric axioms makes its appearance on p. 725 , and the next fifty pages are devoted to a discussion of proofs. This discussion is unavoidably defective.

${ }^{9}$ I wish to thank Alan Schoenfeld for help with the literature on this issue.
} 
formal procedures. Therefore an important component in the teaching of geometry to prospective teachers has to be the fostering of geometric intuition. There is no canonical way to achieve this goal, but $\S 0$ of [WuBraxton] on Experimental geometry offers some concrete suggestions.

At the heart of the general discontent with geometry is the presence of proofs. Since the whole of mathematics is an unending sequence of proofs, ${ }^{10}$ it may come as a surprise to some that the school mathematics curriculum of most states requires proofs only in geometry (if at all). This is the reality of mathematics education at the moment, and this is the reason we take up the discussion of proofs in earnest in this review. The key issue with teachers in geometry is then whether they acquire fluency in writing and explaining proofs. They cannot do that if they fail to recognize that mathematics in general, and geometry in particular, are not only about procedures but also about ideas and reasoning. This message has not been effectively conveyed to prospective teachers in college mathematics courses in general, to say the least (cf. [Wu1] and [Wu5]). Unfortunately, this issue is also ignored in education courses on the teaching of mathematics because mathematical content is not generally considered part of the pedagogical discussion. What makes matters worse in geometry is that most of the geometric proofs that teachers have ever encountered are about utter trivialities (see [Schoenfeld] again). When "proof" becomes synonymous with writing five steps for the explanation of every obvious fact, it is difficult for it to be taken seriously. This perception about proofs is an additional obstacle that college geometry courses must overcome.

In order for teachers to come to understand that proofs are the instrument to establish conviction of why something is true, they must be exposed to the proofs of many nontrivial theorems and be made to struggle with proving some of them. They must also see that proofs are not constructed by an unfathomable formal process that can only be copied and memorized. Rather, proofs are the result of an orderly re-organization of one's thoughts after one has figured out how to reach the desired conclusion by plenty of (chaotic) trials and errors.

For this reason, a college course that gives formal lectures from the beginning and only gives the kind of elegant, streamlined deductions that mathematicians tend to prize would most likely deepen teachers' distrust of proofs. Giving de-constructions of existing proofs and showing how the written version of a proof is often a re-organization of the

\footnotetext{
${ }^{10}$ This is true not only of pure mathematics but of applied mathematics as well. But the discussion of the latter point requires a more sophisticated understanding of what constitues a "proof" in a particular context, so this review will concentrate on pure mathematics.
} 
thought process in reverse order may work better. Beyond such details, there is the global consideration of how to deal with axioms in geometry. For example, is it really necessary to claim absolute rigor by starting with axioms and make endless simple-minded deductions? As before, there is more than one way to confront this issue. Because of the lack of space, only a few possible models will be suggested as references: the geometric portion of [Japan], Appendix D in [Framework], [Lang-Murrow], and $\S 3$ of [Wu-Braxton]. Notice that what these have in common is, in one way or another, a conscientious effort to sidestep proving trivial simple facts and an attendant emphasis on proving more interesting, substantive theorems.

Finally, one must face up to the fact that all high school teachers of geometry should be able to explain to students the main ideas of an axiomatic system. This is because students who go on to study mathematics will encounter axioms at every turn in more advanced work, and because even for students who do not go to college, knowing something about the basic methodology of science and mathematics would round off their education nicely. ${ }^{11}$ The geometric education of prospective teachers therefore should include at least a naive discussion of axioms, consistency, and logical deduction.

Some care needs to be exercised on how to teach axiomatic systems. It seems natural to get teachers accustomed to some proofs first before exposing them to axioms; after all, axioms are generically the organizational afterthought of a mature subject. ${ }^{12}$ However, such pedagogical fine points may be best left to each instructor.

An even-handed discussion of the concept of an axiomatic system with an appropriate amount of illustration of how such a system works, and with due emphasis on its importance but without overselling it in the context of school mathematics requires mathematical sophistication such as only a professional mathematician can give. One would therefore expect that this is where a book such as [Holme] would excel. But this expectation is not met, and the letdown is the more surprising because the statement "we need to do some serious work on foundations" in the preface (p. viii) indicates that the author is well aware of the need to firm up teachers' mathematical fundamentals.

\footnotetext{
${ }^{11}$ Some of them may encounter, for example, popular expositions of special relativity with statements such as "Einstein postulated that the speed of light is constant".

${ }^{12}$ It may be recalled that, for instance, the Eilenberg-Steenrod axioms for homology (1945) came a good fifty years after Poincaré's pioneering work (1895). Nowadays, there are some who can profitably use axiomatics in their research, but for most mathematicians, theorems precede axioms.
} 
The teaching of axiomatic geometry gets off on the wrong foot in Chapter 7 in its discussion of Euclid's and Hilbert's axioms. It lists on p. 167 the five axioms of Euclid without any comments on the odd character of some of the definitions that find their way into these five axioms ${ }^{13}$, or on why five axioms are comically inadequate for the development of the subject. (It also doesn't help to find on p. viii of the preface the statement that "Euclid's original system of axioms and postulates passed remarkably well the test of modern demands to $[$ sic $]$ rigor".) Then without a pause, it blandly summarizes (still on p. 167) Hilbert's axioms in three sentences and states that the existence of this system ensures that "Euclid's postulates can be made to work in a rigorous modern axiomatic setting" (p. 168).

The exposition then forges ahead on these shaky grounds by introducing on the same page (p. 168) the concept of neutral geometry as "a system based on Hilbert's axioms but without the Parallel Postulate", never mind that the reader has no idea what Hilbert's axioms are. Neutral geometry is central in subsequent discussions of various non-Euclidean geometries, yet no more is said about it beyond the fact that it is geometry without the parallel postulate. Instead, the reader is treated to a seven-page account of "Zermelo-Frankel-Skolem axiomatic set theory" and a discussion of Gödel's theorem (pp. 170-176), followed by eighteen pages of axiomatic projective geometry (177-194) and twelve pages on non-Euclidean geometries (pp. 195-207).

This approach to the education of teachers follows the script of the so-called intellectual-trickle down theory ([Wu1]): If prospective teachers learn elegant and advanced mathematics, they would digest it and use it to enhance their high school teaching. The trickle-down theory has not worked so far, and there is no reason to believe that it will work any time soon. ${ }^{14}$ In the case at hand, Holme decides that instead of spending time firming up teachers' dysfunctional knowledge of Euclid, he should spend the time on a little axiomatic set theory, Gödel, projective geometry, and non-Euclidean geometry. But it is no more than a forlorn hope that a short exposure to Zermelo, Gödel, Desargues, and Lobachevsky in succession would inspire prospective teachers to a new level of understanding about what Euclid tried to accomplish with his axioms, why Hilbert had to provide a new foundation, and why it is neither profitable nor realistic to try to attain total rigor at the level of high school geometry. The general reader of [Holme] will continue

\footnotetext{
${ }^{13}$ Recall that these definitions include: 1. A point is that which has no part. 2. A line is breadthless length. 4. A straight line is a line which lies evenly with the points on itself.

${ }^{14}$ On pp. 9-13 of [Wu1], one can find a more extended discussion of why this theory fails.
} 
to be puzzled by Euclid's axiomatic system.

Can teachers learn about proofs or enhance their geometric intuition by reading [Holme]? Not likely. Without attempting to report on 196 pages of mathematics (the length of Part II), we hope an example or two would serve to illustrate this point. Having defined a conic as the graph of a quadratic polynomial in two variables, [Holme] gives a characterization of a non-degenerate conic as the graph of a polynomial whose coefficient matrix has a nonzero determinant. This is Theorem 18 on p. 249. Unfortunately, the reader is not told what a non-degenerate conic is except by inference (p. 237), and even then the definition is not complete because it tacitly assumes the theorem on the reduction of such polynomials to canonical forms. In a footnote (footnote 2 on p. 248), [Holme] says that the proof of Theorem 18 is self-contained because it avoids the use of the reduction theorem, but this assertion is undercut by the very definition of non-degeneracy. One may add that the reduction theorem is also used implicitly in the proof of Theorem 18 more than once (cf. the bottom of p. 250 and also the top of p. 251).

Let us continue a bit with the discussion of conics. [Holme] does try to prove the theorem that conics are exactly the plane sections of a fixed circular cone (Corollary 5 on p. 300). This is a theorem that all geometry teachers ought to know, but [Holme] only proves half of it (every plane section is a conic). ${ }^{15}$ In addition, the proof - couched as it is in the language of projective coordinates and projective equivalence, concepts often troublesome even to undergraduates majoring in mathematics - is hardly designed to reach out to prospective teachers.

If we want to teach teachers about proofs, then we would want them to understand that a proof must start with precise definitions and precise hypotheses (always with the understanding that the precision is appropriate to the given context; see the discussion in $\S \S 2-3$ of [Wu4] for a hint of this delicate issue). When all is said and done, what we want teachers to be absolutely convinced is that, in mathematics, there is no hidden agenda. We would also try to achieve maximum transparency in the arguments because we are constantly maneuvering for credibility. Any exposition that does not meet these basic requirements has little hope of teaching teachers how to write proofs, much less teaching them how to teach their students how to write proofs. It

\footnotetext{
${ }^{15}$ The interesting part of this theorem is not the projective statement that every conic in the plane is projectively equivalent to a plane section, but the Euclidean statement that it is congruent to a plane section.
} 
is fair to say that [Holme] is not succeeding in this task.

But Part II of [Holme] has a more ambitious goal than just educating teachers. It also tries to supply the mathematical underpinnings of the history in Part I. In so doing, it traverses a vast mathematical terrain in 196 pages: projective geometry, non-Euclidean geometry, algebraic geometry of the projective plane, the real number system, elementary theory of fields and constructions by straightedge and compass, Riemannian geometry, fractal geometry, and catastrophe theory. The mere listing of such a large number of topics is already a warning that the author is treading on the dangerous mile-wide-and-inch-deep ${ }^{16}$ territory. Be that as it may, it would still be possible to convey something substantial about these advanced topics if the author would maintain a light touch in the exposition, give careful explanations of a few essential points of each topic, and be very considerate of his audience's mathematical level. It cannot be said that Holme acquits himself well on all counts. Take the simple matter of language: the terms "zero locus" (pp. 145, 250, 270), "polynomials over $K$ " (pp. 319 ff.), "injective mapping" (p. 216), "compactification" (pp. 188-189), and "projective properties" (p. 230) are used without comment. The lack of consideration for his audience extends in many directions, but I have to make do with just one example. Consider the use of "lim" in the first Problem on p. 218: a real number has just been defined as an equivalence class of Cauchy sequences in $\mathbf{Q}$, and not a word is said about the meaning of the "distance" between two real numbers so defined; yet the problem asks for the proof that a Cauchy sequence of real numbers must have a limit. Who in the intended audience could come close to understanding what is being asked?

Every reviewer has to make an obligatory reference to typos and allied irregularities that beset every book. [Holme] has its share of typos, some of them amusing (e.g., "breathless length" on line 16 of p. 69 should be "breadthless length"). It also has quite a few linguistic oddities, such as "stumbling stones" on p. vi (line 14). But instead of giving a list of those, I will conclude by pointing out a few errors that are of mathematical and historical interest.

Page 7 tells us that Babylon was inside the present city of Baghdad; it was in fact outside present day Al Hillah, some sixty miles south of Baghdad. ${ }^{17}$ On p. 69 it is stated that "Questioning the truth of the

\footnotetext{
${ }^{16}$ I am happy to borrow this famous phrase of Bill Schmidt, who used it to describe the school mathematics curriculum in the U.S.

${ }^{17} \mathrm{~A}$ map of the archaeological sites can be found on p. 109 of [Robson 2].
} 
assertions upon which Euclidean Geometry rested came, in the end, to be regarded as heretics" (lines 3-4), and the case of Giordano Bruno being burnt at the stake is brought up in the same context on p. 169 to reinforce this idea that "Euclidean Geometry had, so to say, been canonized by the Catholic Church" (lines 13-14). We know that the Church persecuted those who challenged Ptolemy, but now we are told, contrary to everything we have come to know about the Middles Ages, that the Church was just as zealous in defending Euclid. Without the support of unimpeachable evidence, such a bald assertion lacks credibility. On p. 138, we are told that Desargues' Theorem is contained in Desargues' 1639 Brouillon project, but it was actually published by Abraham Bosse in an appendix to the latter's 1648 treatise, Manière universelle de M. Desargues ... ([Field-Gray]). Still on p. 138, axiomatic geometry is said to be "a comparatively small field" (line 10), but on p. viii of the preface, there is an almost opposite statement that "[axiomatic projective geometry] is an extensive field in itself" (line -8). The definitions of "congruence" and "similarity" on p. 213 are incorrect: the author forgot about congruences and similarities that are orientation-reversing. On p. 235, one finds a dismissive statement that the study of the "so-called non-Desargian[sic] planes is an exotic interest pursued by some mathematicians". However, the content of $\S 8.2$ on pp. 182-184 is about the search for projective planes whose order is not a prime power, i.e., non-Desarguesian planes, and it is stated on p. 182 that this is an "interesting open geometric problem". Finally, Lorenzo Mascheroni was credited with the discovery that all points in the plane obtainable by straightedge and compass constructions can be obtained by compass alone (p. 330). But for over seventy-five years, Scandinavian scholars have known that the Danish geometer Georg Mohr had anticipated Mascheroni by 125 years! ([Eves])

As a popular account of one area of mathematics, [Holme] has some attractive features. Despite its sometimes opaque explanations and its occasional mathematical and historical errors, it provides a sense of the drama of mathematical developments and their role in world culture. This could be a valuable contribution. But the book fails in its efforts to provide a basis for courses for prospective geometry teachers. Given the dismal state of teacher education, and the crying need for well thought out textbooks, we need something far better. If the professional development of mathematics teachers were stronger than it is, [Holme] might be counted upon as one source of material among many, and teachers would be better equipped to discern its 
potential uses and its faults. But in the United States today, a book like [Holme] is likely to do more harm than good.

\section{REFERENCES}

[Beckmann] S. Beckmann, Mathematics for Elementary Teacher, Volume 2: Geometry, Addison Wesley, 2003.

[Borel] A. Borel, Twenty-five years with Nicolas Bourbaki, Notices Amer. Math. Soc., 45 (1998), 373-380.

[Deakin 1] M. A. B. Deakin, Hypatia and her mathematics, Amer. Math. Monthly, 101 (1994), 234-243.

[Deakin 2] M. A. B. Deakin, Review of "Hypatia of Alexandria" by Maria Dzielska, Amer. Math. Monthly, 103 (1996), 83-87.

[Eves] H. Eves, A Survey of Geometry, Volume 1, Allyn and Bacon, 1972.

[Field-Gray] J. V. Field and J. J. Gray, The Geometric Work of Girard Desargues, SpringerVerlag, 1987.

[Framework] Mathematics Framework for California Public Schools, Kindergarten Through Grade Twelve, 2000 revised edition, California Department of Education, 2000. Also http://www.cde.ca.gov/cdepress/math.pdf

[Heath 1] T. L. Heath, Euclid: The Thirteen Books of the Elements, Volumes 1-3, Dover, 1956.

[Heilbron] J. L. Heilbron, Geometry Civilized: History, Culture, and Technique, Oxford University Press, 1998.

[Hilbert-CV] D. Hilbert and S. Cohn-Vossen, Geometry and the Imagination, Chelsea, 1952. (Amer. Math. Soc. reprint, 2003.)

[Japan] Japanese Grade 7 Mathematics, Japanese Grade 8 Mathematics, Japanese Grade 9 Mathematics, University of Chicago Mathematics Project, University of Chicago, 1992.

[Lang-Murrow] S. Lang and G. Murrow, Geometry, 2nd edition, Springer-Verlag, 1998.

[MET] The Mathematical Education of Teachers, CBMS Issues in Mathematics Education, Volume 11, American Mathematical Society, 2001.

[NCT] What Matters Most: Teaching for America's Future, Report of the The National Commission on Teaching and America's Future, September, 1996.

[Osserman] R. Osserman, Are proofs in high school geometry obsolete? Preprint.

[Robson 1] E. Robson, Neither Sherlock Holmes nor Babylon: A reassessment of Plimpton 322, Historia Mathematica, 28 (2001), 167-206.

[Robson 2] E. Robson, Words and pictures: New light on Plimpton 322, Amer. Math. Monthly, 109 (2002), 105-120.

[Russell] B. Russell, The Autobiography of Bertrand Russell: 1872 to World War I, (paperback edition), Bantam Books, 1968.

[Schoenfeld] A. H. Schoenfeld, When good teaching leads to bad results: The disasters of "well-taught" mathematics courses, Education Psychologist, 23 (1988) 145-166.

[Serra] M. Serra, Discovering Geometry, 2nd edition, Key Curriculum Press, 1997.

[Wu1] H. Wu, On the education of mathematics majors, Contemporary Issues in Mathematics Education, edited by E. Gavosto, S. G. Krantz, and W. G. McCallum, MSRI Publications, Volume 36, Cambridge University Press, 1999, 9-23. Also http://math.berkeley.edu/ wu/

[Wu2] H. Wu, Professional development of mathematics teachers, Notices Amer. Math. Soc. 46 (1999), 535-542. Also http://www.ams.org/notices/199905/fea-wu.pdf

[Wu3] H. Wu, Preservice professional development of mathematics teachers, (March 1999), http://math.berkeley.edu/ wu/

[Wu4] H. Wu, What is so difficult about the preparation of mathematics teachers? http://math.berkeley.edu/ wu/ 
[Wu5] H. Wu The issue of mathematical substance in the professional development of mathematics teachers, in preparation.

[Wu-Braxton] H. Wu and B. Braxton, Content Statements for Elementary Geometry, at http://tepd.ucop.edu/tepd/cpdi/mpdi_home.html (click on "Revised Elementary Geometry August 2002" near the bottom of the webpage).

Department of Mathematics, University of California, Berkeley, CA 94720-3840, WU@MATH.BERKELEY.EDU 